\title{
Assistência Pré- Natal: Uma Avaliação do Serviço Público de Saúde no Atendimento às Gestantes no Município de Icó- CE
}

\author{
Vanessa Machado Custódio Dantas ${ }^{1}$, Raimundo Tavares de Luna Neto ${ }^{2 *}$, Kerma Márcia de Freitas ${ }^{3}$, Olga Feitosa Braga \\ Teixeira ${ }^{4}$, Francinete Alves Felipe ${ }^{5}$, Alan Felipe Oliveira de Alencar ${ }^{6}$, Cleciana Alves Cruz ${ }^{7}$, Úrsula Hérica dos Santos \\ Moura $^{8}$, Natália Bastos Ferreira Tavares ${ }^{9}$
}

Resumo: Tendo em vista as inúmeras transformações biopsicossociais que a mulher apresenta durante o período gravídico, o pré-natal vem como o objetivo de assegurar um desenvolvimento de uma gestação segura e livre de riscos, bem como, um parto e pós-parto saudáveis. A assistência a gestante no acompanhamento pré-natal, no entanto, consiste na junção de ações e instrumentos que unidos exercem medidas de assisti-las em todas as suas necessidades, assim como, na realização de condutas preventivas, educativas e curativas. A presente pesquisa tem por objetivo avaliar a qualidade da assistência prestada as gestantes do município de Icó- CE, com foco na estrutura, no processo e nos resultados da Unidade Básicas de Saúde- UBS. Sendo composta por duas fases, a primeira uma pesquisa do tipo exploratório descritiva, transversal com abordagem quantitativa e a segunda do tipo documental, devido a utilização de dados secundários. O estudo foi realizado nas 08 equipes de saúde da família da zona urbana do município e na Secretaria de Saúde da referida cidade. Foi utilizado o instrumento de Avaliação da Qualidade da Assistência Pré-Natal criado por Rocha (2011). Os dados foram organizados em tabelas e após serem tabulados foram confrontados com a literatura. Para realização deste estudo foram observados os pressupostos da resolução 466/12 do Conselho Nacional de Saúde do Ministério da Saúde (CNS MS) que do qual dispõe sobre pesquisa com seres humanos. Após a análise dos dados no que tange à estrutura das unidades da zona urbana da cidade, pode-se observar que as mesmas atendem aos requisitos mínimos e considerados indispensáveis para que esta assistência possa ocorrer com qualidade, porém há ainda os vieses e pontos falhos que ainda podem interferir na qualidade destes atendimentos e que precisam de atenção. Com relação aos indicadores, apesar de não terem como ter sido usado todos aqueles disponíveis pelo MS, pelo fato da indisponibilidade de dados do SISPRENATAL e por este ainda estar em processo de adaptação, nem todos os utilizados refletiram um resultado satisfatório, mostra que há pontos frágeis que também necessitam de de cuidado.

Palavras- chave: Assistência integral à saúde. Atenção Primária à Saúde. Cuidado Pré- Natal.

\section{Assistência Pré- Natal: Uma Avaliação Do Serviço Público De Saúde No Atendimento As Gestantes No Município De Icó- Ce}

Resumo: Tendo em vista as inúmeras transformações biopsicossociais que a mulher apresenta durante o período gravídico, o pré-natal vem como o objetivo de assegurar um desenvolvimento de uma gestação segura e livre de riscos, bem como, um parto e pós-parto saudáveis. A assistência a gestante no acompanhamento pré-natal, no entanto, consiste na junção de ações e instrumentos que unidos exercem medidas de assisti-las em todas as suas necessidades, assim como, na realização de condutas preventivas, educativas e curativas. A presente pesquisa tem por objetivo avaliar a qualidade da assistência prestada as gestantes do município de Icó- CE, com foco na estrutura, no processo e nos resultados da Unidade Básicas de Saúde- UBS. Sendo composta por duas fases, a primeira uma pesquisa do tipo exploratório descritiva, transversal com abordagem quantitativa e a segunda do tipo documental, devido a utilização de dados secundários. O estudo foi realizado nas 08 equipes de saúde da família da zona urbana do município e na Secretaria de Saúde da referida cidade. Foi utilizado o instrumento de Avaliação da Qualidade da Assistência Pré-Natal criado por Rocha (2011). Os dados foram organizados em tabelas e após serem tabulados foram confrontados com a literatura. Para realização deste estudo foram observados os pressupostos da resolução 466/12 do Conselho Nacional de Saúde do Ministério da Saúde (CNS MS) que do qual dispõe sobre pesquisa com seres humanos. Após a análise dos dados no que tange à estrutura

\footnotetext{
$\overline{1,3,7,8}$ Enfermeiro(as). Faculdade Vale do Salgado-FVS, Icó-Ce.

${ }^{2,9}$ Enfermeiro. Programa de Pós Graduação em Ciências da Saúde pela Faculdade de Medicina do ABC. Docente da Universidade Regional do Cariri (URCA) / Unidade Descentralizada de Iguatu-CE. Faculdade Vale do Salgado-FVS.

${ }^{4}$ Enfermeira. Departamento de Enfermagem, Universidade Federal de Campina Grande, Campus Cajazeiras.

${ }^{5}$ Residente em Saúde da Família e Comunidade pela Escola de Saúde Pública do Ceará/MS.

${ }^{6}$ Faculdade de Medicina Estácio de Sá- Juazeiro do Norte.

* Autor correspondente: Raimundo Tavares de Luna Neto. E-mail: duquinhatavares@gmail.com
} 
das unidades da zona urbana da cidade, pode-se observar que as mesmas atendem aos requisitos mínimos e considerados indispensáveis para que esta assistência possa ocorrer com qualidade, porém há ainda os vieses e pontos falhos que ainda podem interferir na qualidade destes atendimentos e que precisam de atenção. Com relação aos indicadores, apesar de não terem como ter sido usado todos aqueles disponíveis pelo MS, pelo fato da indisponibilidade de dados do SISPRENATAL e por este ainda estar em processo de adaptação, nem todos os utilizados refletiram um resultado satisfatório, mostra que há pontos frágeis que também necessitam de de cuidado.

Palavras- chave: Assistência integral à saúde. Atenção Primária à Saúde. Cuidado Pré- Natal.

\section{Introdução}

A gestação é considerada um fenômeno fisiológico pleno de novas e profundas transformações na vida da mulher e deve ser visto pelas gestantes e equipe de saúde como uma fase integrante de uma experiência de vida saudável que envolve naturalmente mudanças nos aspectos físico, social e emocional. Para tanto, a Unidade Básica de Saúde (UBS) é considerada o principal ponto referencial de assistência prestada a estas mulheres, pois é nas consultas que se realizam todas as ações e orientações consideradas importantes para a mãe e os cuidados pós-parto, tendo então o profissional a importante missão de proporcionar um acolhimento e uma assistência satisfatória às mesmas (BRASIL 2012a)

Segundo Rocha (2011), a qualidade de saúde das gestantes e conceptos estão intimamente ligados a qualidade da assistência prestada no pré-natal, pois este diminui significativamente índices indesejáveis pertinentes a saúde de ambos, durante o período gravídico ou mesmo após o parto. Sendo a atenção a gestante, em especial, uma necessidade incorporada na Estratégia de Saúde da Família (ESF), com a finalidade de assegurar uma qualidade de saúde satisfatória para os envolvidos.

Corroborando para o entendimento, Costa et al. (2010) afirmam que, a qualificação no processo de assistência ao pré-natal está centrada na atuação profissional, bem como nos programas infundidos pelo Ministério da Saúde (MS) com o objetivo de diminuir das taxas de morbimortalidade materna e perinatal.

Sendo assim, Zanchi et al. (2013) afirmam que as pesquisas direcionadas na avaliação da qualidade da assistência a saúde contribuem significativamente na identificação das problemáticas enfrentadas pelo serviço, bem como, orientam os gestores e profissionais a terem conhecimento da atual situação e na tomada de decisões cabíveis a cada caso.

Vislumbrando pois, tamanho significado desta assistência, tem-se a necessidade de uma constante avaliação da mesma em busca de achados que possam, portanto, serem melhorados, minimizando assim, riscos relacionados à saúde materno-neonatal. Nesse sentido, este estudo visa responder as seguintes problemáticas: Será que a assistência prestada às mulheres no seu período 
gravídico na cidade de Icó-CE está sendo desenvolvida de maneira satisfatória? Será que tanto os recursos humanos quanto materiais estão disponíveis em sua totalidade nas UBS? Os indicadores estão favoráveis, mediante as ações que estão sendo realizadas nas unidades? Dentre estes questionamentos parte então a iniciativa de se avaliar de maneira integral esta assistência.

Com o intuito de fornecer informações aos profissionais responsáveis pelas unidades e gestores envolvidos, este estudo tem a magnitude de servir como fonte de informação útil na avaliação da assistência prestada as gestantes da referida cidade, bem como consequentemente, incentivar a constante reavaliação das ações por parte de ambos a partir da coleta de dados e de sua tabulação, buscando uma melhoria dentro de seus limites de atuação, trazendo assim uma procura pela qualidade não só em números, mas no que tange os recursos mínimos exigidos para uma assistência qualificada.

Considerando o exposto ressalta-se que o estudo tem por objetivo geral avaliar a qualidade da assistência prestada as gestantes do município de Icó- CE, com foco na estrutura, no processo e nos resultados das UBS.

Para tanto, o presente trabalho ostenta quão importante é a avaliação daquilo que se é necessário na assistência pré-natal, pois as ausências destes recursos refletem preocupação no que se referem à qualidade do serviço, sendo a assistência em sua totalidade se iniciando desde o adentrar da mulher na unidade ao atendimento propriamente dito, assim como os seguimentos que se fazem necessários ao bom funcionamento desta assistência. Espera-se que o mesmo possa contribuir no ampliar de conhecimento acerca do estudo exposto e possa ser fonte de motivação para posteriores estudos relacionada a esta temática.

\section{Método}

A presente pesquisa é do tipo exploratório descritiva, transversal com abordagem quantitativa e do tipo documental, devido à utilização de dados secundários.

Os cenários de investigação desta pesquisa foram as UBS da zona urbana e a Secretaria de Saúde do município de Icó-CE

A pesquisa em sua primeira fase foi constituída pelas 08 (oito) equipes das UBS da zona urbana da cidade, sendo elas: Alto Manoel Mariano I e II, Centro, Cidade Nova I e II, Gama, São Geraldo e São Vicente de Paulo. Sendo as mesmas selecionadas para a realização desta pesquisa pelo fato de se apresentarem em uma área de abrangência central.

Os dados foram coletados junto às enfermeiras nas UBS em sua primeira fase, fazendo uso do instrumento que continha questões relacionadas à estrutura, aos recursos e aos materiais disponíveis na unidade para realização da assistência pré-natal. Já a segunda fase foi constituída por uma coleta de dados diretamente da Secretaria Municipal de Saúde da cidade, mais precisamente junto a 
Coordenação de Atenção Básica, com dados pertinentes ao pré-natal de 01 de Janeiro a 30 de Junho de 2014.

Utilizou-se como instrumento de coleta de dados o Instrumento de Avaliação da Qualidade da Assistência Pré-Natal criado por Rocha (2011), validado no período de realização de seu estudo, no período de 2009 a 2011.

A técnica de coleta de dados se configurou em duas etapas, sendo elas:

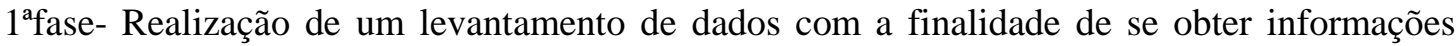
relacionadas à estrutura física da unidade, aos recursos, bem como também, ao fluxo de atividades relacionadas ao atendimento das gestantes.

$2^{\mathrm{a}}$ fase- Nesta fase, os dados foram obtidos nos bancos do SISPRENATAL e Sistema de Informação sobre Nascidos Vivos (SINASC) sendo estes relacionados a informações referentes ao processo, resultado e impacto da assistência as gestantes.

Na primeira fase os dados foram analisados e interpretados, sendo contemplada a planta física, os recursos humanos, os recursos materiais, o apoio laboratorial, a disponibilidade de medicamentos essenciais, funcionamento do sistema de referência e contra referência e os instrumentos registros e para esta avaliação houve uma pontuação para qualificação da assistência, sendo: 0 (zero) ponto para insatisfatório ou inexistente, 05 (cinco) pontos quando fosse parcialmente atendido na unidade, e 10 (dez) pontos quando o quesito fosse totalmente atendido.

Obtendo-se ao final desta fase um total máximo para cada requisito de: Planta física (máximo 70 pontos); Recursos humanos (máximo 80 pontos); Recursos materiais (máximo 140 pontos); Apoio laboratorial (máximo 110 pontos); Medicamentos essenciais (máximo 100 pontos); Referência e contra referência (máximo 20 pontos) e Instrumentos de registro (máximo 30 pontos). Assim sendo, a avaliação final foi realizada de acordo com a autora do instrumento, com a soma total de pontos obtidos, com 550 no máximo, sendo as unidades classificadas em ótimas (90\% a 100\% do total de pontos); satisfatórias ( $75 \%$ a $89,9 \%)$; precárias (50\% a 74,9\%) ou insuficientes (49,9\% e menos).

As Unidades foram distribuídas na tabela por ordem alfabética e recebidas as pontuações correspondentes das quais seus coordenadores no momento da pesquisa assinalaram como sendo quais as condições destes requisitos nas unidades: se faltosos, parcialmente faltosos ou existentes.

Bem como, na segunda fase deste estudo houve o levantamento de dados através do sistema do SISPRENATAL e SINASC junto a SMS, havendo logo em seguida a análise e interpretação fazendo-se uso das fórmulas. Posteriormente os mesmos foram agrupados em classes para facilitar a tabulação e para posterior confronto de resultados, bem como, a discussão dos mesmos.

Foram utilizados regra de três simples para relacionar valores e classificar as unidades em sua primeira fase, bem como, foi utilizado os cálculos disponíveis pelo MS para obter informações em forma de percentual dos indicadores na segunda fase. 
Em todas as etapas da pesquisa foram obedecidas conforme manda a resolução de $n^{\circ} 466 / 12$ do Conselho Nacional de Saúde e Ministério da Saúde, norma que regulamenta a pesquisa envolvendo seres humanos, sendo aplicado aos profissionais coordenadores da UBS sob assinatura do Termo de Consentimento Livre e Esclarecido (TCLE) e sob consentimento do Secretário de Saúde através da assinatura do termo de anuência, bem como, através do Termo de Fiel Depositário assinado pela coordenação de AB. Esta resolução dispõe sobre diretrizes e normas reguladoras de pesquisa envolvendo seres humanos. Incorporando os quatro referenciais da bioética: a autonomia, a não maleficência, beneficência e justiça. Respeitando a dignidade humana, sobretudo os participantes da pesquisa (BRASIL, 2012b).

\section{Análise dos dados}

Neste capítulo, são apresentados os resultados obtidos mediante a análise dos dados, seguindo os objetivos propostos neste estudo. Foram analisados todos os dados obtidos juntos as responsáveis pela administração das 08 equipes de saúde da família do município de Icó- CE. Ressaltando, sobretudo os quesitos que mais se destacaram na pesquisa, bem como, sua classificação final.

TABELA I. Distribuição da pontuação obtida em cada equipe acerca dos requisitos mínimos e indispensáveis das ESF da zona urbana do município de Icó-CE

\begin{tabular}{cccccccccc} 
& $\begin{array}{c}\text { Máximo } \\
\text { de } \\
\text { pontos }\end{array}$ & $\begin{array}{c}\text { ESF } \\
\mathbf{0 1}\end{array}$ & $\begin{array}{c}\text { ESF } \\
\mathbf{0 2}\end{array}$ & $\begin{array}{c}\text { ESF } \\
\mathbf{0 3}\end{array}$ & $\begin{array}{c}\text { ESF } \\
\mathbf{0 4}\end{array}$ & $\begin{array}{c}\text { ESF } \\
\mathbf{0 5}\end{array}$ & $\begin{array}{c}\text { ESF } \\
\mathbf{0 6}\end{array}$ & $\begin{array}{c}\text { ESF } \\
\mathbf{0 7}\end{array}$ & $\begin{array}{c}\text { ESF } \\
\mathbf{0 8}\end{array}$ \\
\hline Planta física & 70 & 70 & 70 & 70 & 45 & 40 & 55 & 70 & 50 \\
\hline $\begin{array}{c}\text { Resursos } \\
\text { humanos }\end{array}$ & 80 & 80 & 70 & 75 & 80 & 75 & 70 & 75 & 80 \\
\hline $\begin{array}{c}\text { Recursos } \\
\text { materiais }\end{array}$ & 140 & 140 & 130 & 140 & 130 & 135 & 140 & 140 & 135 \\
\hline $\begin{array}{c}\text { Apoio } \\
\text { laboratorial } \\
\text { mínimo }\end{array}$ & 110 & 105 & 100 & 110 & 95 & 100 & 80 & 110 & 100 \\
\hline $\begin{array}{c}\text { Medicamentos } \\
\text { essenciais }\end{array}$ & 100 & 90 & 90 & 90 & 75 & 80 & 90 & 90 & 90 \\
\hline $\begin{array}{c}\text { Sistema de } \\
\text { referência e } \\
\text { contra- } \\
\text { referência }\end{array}$ & 20 & 20 & 15 & 15 & 15 & 15 & 15 & 15 & 15 \\
\hline $\begin{array}{c}\text { Instrumentos } \\
\text { de registro }\end{array}$ & 30 & 30 & 30 & 30 & 30 & 30 & 30 & 30 & 30 \\
\hline $\begin{array}{c}\text { Total dos } \\
\text { pontos }\end{array}$ & 550 & 535 & 505 & 530 & 470 & 475 & 480 & 530 & 500 \\
\hline $\begin{array}{c}\text { analisados } \\
\text { FONTE: Dados da pesquisa }\end{array}$ & & & & & & & & \\
\hline
\end{tabular}


Verificou-se na TABELA I, que de 550 pontos máximos presentes no instrumento de coleta de dados, o valor mínimo obtido neste estudo foi de 470 na ESF de no 04 e o máximo foi o de 535 na ESF de $\mathrm{n}^{\circ}$ 01. Sendo esta uma tabela inicial que facilita a organização dos dados para posteriores cálculos mais detalhados.

Rocha (2011), autora do instrumento de coleta de dados, destaca que o instrumento utilizado aqui, avalia na estrutura a existência ou não de itens considerados indispensáveis para a consulta prénatal, tendo a ciência de que o mesmo não avalia a questão do acolhimento para com a paciente, bem como o vínculo existente entre o profissional e a gestante.

Diante disto, é importante salientar que ao dados aqui coletados não incluem a maneira como as gestantes são atendidas, sendo este um ponto importante ainda a ser analisado em posteriores pesquisas, pois a busca pela melhor assistência esta também intimamente relacionada com a qualidade do atendimento prestado.

Um importante aspecto levantado por Polgliane et al. (2014) é que a prática de se avaliar os serviços de saúde devem ser constantes, pois estes tem o propósito de se levantar os pontos que merecem mais atenção afim de garantir os critérios mínimos exigidos na assistência.

Sendo assim, após a tabulação dos dados pôde-se verificar que alguns requisitos tidos como essenciais para a prestação de um serviço qualificado estão faltosos ou inadequados. Havendo a necessidade então que os profissionais tenham conhecimento destas fragilidades para que se possam programar novas estratégias ou mesmo buscar auxílio aos gestores responsáveis.

Com esta maneira de representação, podem-se identificar alguns pontos fragilizados, tais como, no sistema de contra-referência, pois este por quase toda a totalidade foi citado como falho, bem como, a questão estrutural de algumas unidades das quais as equipes se fazem presentes, podendo-se analisar ainda que existe um fator positivo forte que chega a atingir $100 \%$ das unidades como os instrumentos de registro.

Algumas unidades, por sua vez, apresentam-se em estruturas impróprias, em prédios não padronizados pelo MS, sendo este o motivo de algumas equipes não terem todo o aparato suficiente e apresentarem resultados não tão satisfatórios quanto as demais unidades. As equipes que se apresentam nestas estruturas não padronizadas enfrentam um desafio contínuo, pois tentam diariamente prestar seus serviços da melhor forma possível, porém dentro de limitações que não os permite ir além do esperado em suas ações, pelo fato de muitas vezes a estrutura não favorecer a realização de determinadas atividades.

Polgliane et al. (2014) em sua pesquisa, afirmam que estudos acerca da temática aqui exposta são peças que podem mudar o rumo de muitas nações, pois identificam pontos e propõem estratégias para sua mudança. Relata ainda que é relevante a avaliação do pré-natal, principalmente em países com amplas desigualdades regionais, econômicas, sociais e de acesso aos serviços de saúde. 
TABELA II. Classificação geral da estrutura das unidades da zona urbana do município de Icó- CE.

\begin{tabular}{lccc}
\hline & $\begin{array}{c}\text { PONTUAÇÃO } \\
\text { APURADA }\end{array}$ & $\%$ & CLASSIFICAÇÃ $*^{*}$ \\
\hline ESF 01 & 535 & 97 & Ótima \\
\hline ESF 02 & 505 & 92 & Ótima \\
\hline ESF 03 & 530 & 96 & Ótima \\
\hline ESF 04 & 470 & 85 & Satisfatória \\
\hline ESF 05 & 475 & 86 & Satisfatória \\
\hline ESF 06 & 480 & 87 & Satisfatória \\
\hline ESF 07 & 530 & 96 & Ótima \\
\hline ESF 08 & 500 & 90 & Ótima \\
\hline
\end{tabular}

Fonte: Dados da pesquisa

*Ótimas (90\% a 100\%); satisfatórias (75\% a 89,9\%); precárias (50\% a 74,9\%); ou insuficientes $(49,9 \%$ e menos).

Nesta tabela quanto a classificação pode-se verificar que das 08 (oito) equipes estudadas 05 (cinco) delas pôde ser classificadas como ótimas e 03 (três) como satisfatórias, mediante a utilização do instrumento de coleta de dados usado como referência.

Sendo importante ainda ressaltar mesmo diante de resultados bastante otimistas de uma forma geral, que muitas unidades necessitam de atenção quanto a recursos mínimos faltosos, sendo eles os mais importantes: recursos materiais, de espaço e documental, porém que refletem uma importância impar para a atenção as gestantes.

Paris; Pelloso; Martins (2013) relatam que pesquisar a qualidade da assistência ao pré-natal é uma ação de grande relevância, sendo considerada um passo indispensável para o conhecimento das limitações e deficiências das UBS.

É possível perceber tamanha importância de tais estudos relacionados a esta temática, pois estes refletem conhecimentos acerca da situação atual e consequentemente instiga os profissionais a sempre buscarem novas ações que podem ser idealizadas e posteriormente realizadas.

Visto que ainda é necessário estar ciente que a avaliação da assistência não se limita somente nos muros do local de realização das consultas, mas também, no atendimento em si. Avaliar o prénatal requer bastante atenção e cuidado, pois este leva em consideração infinitos pontos a serem vistos e percebidos pelos próprios profissionais. 
TABELA III- Avaliação dos indicadores do primeiro semestre de 2014 do município de Icó-CE.

\begin{tabular}{l|c}
\multicolumn{1}{c|}{ INDICADORES } & $\%$ \\
\hline $\begin{array}{l}\text { Número de Gestantes que realizaram a primeira consulta pré- natal } \\
\text { até o quarto mês gestacional em relação à população- alvo. }\end{array}$ & 51,8 \\
\hline $\begin{array}{l}\text { Percentual de gestantes que realizaram no mínimo seis consultas } \\
\text { pré- natal. }\end{array}$ & 23,7 \\
\hline Percentual de gestantes que receberam imunização antitetânica. & $69,63 \%$ \\
\hline $\begin{array}{l}\text { Percentual de recém- nascidos com diagnóstico de sífilis congênita } \\
\text { em relação ao total de rerém- nascidos do município. }\end{array}$ & 0 \\
\hline $\begin{array}{l}\text { Percentual de recém- nascidos com diagnóstico de tétano neonatal } \\
\text { em relação ao total de recém- nascidos do município }\end{array}$ & 0 \\
\hline $\begin{array}{l}\text { Razão da mortalidade materna do município comparada a do ano } \\
\text { anterior. }\end{array}$ & 0 \\
\hline $\begin{array}{l}\text { Coeficiente de mortalidade neonatal no município comparado ao } \\
\text { do ano anterior. }\end{array}$ & $20,32 \%$ \\
\hline
\end{tabular}

Fonte: SISPRENATAL; SINASC

Os números aqui expostos foram obtidos mediante a utilização dos cálculos disponibilizados pelo MS e utilizando-se os valores fornecidos pelo SISPRENATAL e SINASC, onde após a coleta dividia-se o valor pelo número total de gestantes ou de nascidos vivos, isto a depender do calculo e multiplicando então por 100 ou 1000, este também a depender da fórmula utilizada.

No transcorrer desta fase da pesquisa, teve-se ciência no momento da coleta de dados, que as informações do software SISPRENATAL do ano de 2013 do referido município de estudo, apresentava-se indisponível para que se pudessem coletar os dados solicitados dos indicadores. Por este motivo, foi utilizado assim, dados referentes ao primeiro semestre de 2014 , onde ainda somente 07 (sete) dos 10 (dez) indicadores do MS foram utilizados, pelo fato de alguns números se apresentarem indisponíveis, sendo eles: Percentual de gestantes que realizaram no mínimo seis consultas pré-natais e consulta de puerpério; Percentual de gestantes que realizaram no mínimo seis consultas de pré-natal e todos os exames básicos e o Percentual de gestantes que realizaram no mínimo seis consultas pré-natal, a consulta de puerpério, todos os exames básicos, o teste anti-HIV e a imunização antitetânica. Para tanto esta fase da pesquisa foi composta por dados das unidades do município como um todo e não somente da zona urbana, correspondendo então a um total de 20 UBS.

Este indicadores aqui construídos, ainda que sendo ditos do MS, não podem ser considerados fidedignos neste estudo, pois o programa do qual foi colhido boa parte destas informações estava momentaneamente passando por um processo de adaptação ou aperfeiçoamento, não nos fornecendo dados reais, segundo informações colhidas na referida secretaria de saúde da cidade. 
No primeiro semestre de 2014 o município de Icó contou com 471 gestantes cadastradas no programa SISPRENATAL, sendo que destas apenas 112, ou seja, 23,77\% realizaram 6 (seis) ou mais consultas, segundo o sistema citado anteriormente. Outro dado ainda bastante pertinente está relacionado à quantidade de gestantes que realizaram consultas até $12^{\mathrm{a}}$ semana gestacional sendo que deste total apenas 244 , ou seja, $51,80 \%$ obtiveram esta assistência.

Estes dados exprimem que ainda há uma necessidade de se buscar conhecer o que de fato ocorre para que tais dados ainda estejam abaixo do esperado, logo que reflete de uma maneira negativa a assistência prestada para com estas mulheres, no que tange as consultas e sua frequência à unidade.

Como já abordado por Brasil (2012a) a assistência pré-natal realizada de forma adequada com seus respectivos componentes de detecção e a intervenção precoce das situações de risco, bem como um sistema ágil de referência hospitalar, sistema de regulação, regulação de leitos obstétricos, vinculação da gestante à maternidade, além da qualificação da assistência ao parto, são importantes determinantes dos indicadores de saúde onde estão diretamente relacionados à mãe e ao bebê e têm o potencial de diminuir as principais causas de mortalidade materna e neonatal.

Andreucci e Cecatti (2011) destaca que independentemente da forma como a unidade registre os dados de seus atendimentos, os mesmos devem contemplar de forma efetiva a documentação. Sendo que são estes documentos que alimentam os sistemas responsáveis pela avaliação do processo da assistência prestada e evitam a fragmentação do atendimento das usuárias nos diferentes níveis de atenção.

Quando se diz que deverá se avaliar o processo este está relacionado a buscar informações referentes às ações desenvolvidas nos serviços de saúde, essencialmente no que tange a atuação profissional no cuidado do binômio saúde/doença da população.

O SISPRENATAL, portanto foi o instrumento que permitiu o acompanhamento da adesão ao PHPN em nível nacional, desde seu cumprimento de metas até as estatísticas de saúde maternoinfantil. Ainda nesta mesma linha de considerações Andreucci; Cecatti (2011) após a realização de seus estudos acerca do programa SISPRENATAL, afirma na sua presente revisão sistemática que o referido sistema, parece apresentar falhas de registro dos procedimentos mínimos recomendados na atenção das gestantes, em comparação com outras fontes de informação. Outro aspecto levantado é que há a necessidade de que haja sempre uma comparação entre este sistema e outras fontes de informações de forma simultânea e em uma mesma população para que se possa verificar como estes estão sendo alimentados.

O SISPRENATAL ainda é novo e tem por objetivo cadastrar as gestantes que tem acesso a rede básica de saúde para a realização do acompanhamento pré-natal por intermédio do acesso à base do Sistema de Cadastramento de Usuários do SUS (CADSUS), o mesmo, no entanto disponibiliza informações que podem ser vistas em tempo real e que podem servir de base para se analisar os indicadores da RC e todo o transcorrer desta assistência. Além disso, o preenchimento da Ficha de 
Cadastro da Gestante no SISPRENATAL deve possibilitar a todo o apoio a gestante as consultas à vinculação da gestante ao local do parto (BRASIL, 2012a).

Outro dado importante que foi disponibilizado através do SISPRENATAL foi a relação da quantidade de gestantes que realizaram os exames básicos até o $1^{\circ}$ trimestre gestacional, sendo de somente 204 gestantes para o total de 471, correspondendo a apenas 43,31\%. Um resultado bastante preocupante, pois os exames são considerados os requisitos básicos para se proporcionar uma gestação saudável, bem como um parto e pós parto seguro, além de evitar possíveis agravos.

Através do SINASC puderam-se também levantar alguns dados referentes aos indicadores de impacto tais como razão da mortalidade materna, bem como, coeficiente de mortalidade neonatal total do município, sendo o primeiro zerado no primeiro semestre de 2014 e o segundo obteve 10 de um total de 492 nascidos vivos. De 0 (zero) a 6 (seis) dias o município teve 07 (sete) óbitos e de 7 (sete) a 27 (vinte e sete) dias obteve 03 (três), perfazendo um total de 10 (dez) no município como um todo. Para tanto a partir do calculo realizado utilizando-se o numero de óbitos de RN de 0 a 27 e dividindo pelo numero de nascidos vivos e posteriormente multiplicando por 1.000 chegou-se ao resultado de $20,32 \%$ do total de nascidos vivos.

As mortes maternas são classificadas em obstétricas diretas e indiretas. Sendo a primeira relacionada a complicações surgidas durante o período gestacional, parto ou puerpério e as indiretas relacionadas a patologias já existentes na mesma, que se desenvolveram na gestação ou que também se acentuaram ou agravaram neste período decorrente do processo fisiológico a que a gestação se configura (BRASIL, 2012a).

No que tange a mortalidade neonatal, a melhoria do cuidado na assistência ao pré-natal pode ser considerada uma das mais importantes metas a serem alcançadas em termos de saúde pública devido a sua capacidade de redução dos fatores desencadeantes da sua morbimortalidade (VETTORE et al. 2013).

Considerando a imunização antitetânica, o presente estudo encontrou que o percentual de gestantes que receberam imunização antitetânica correspondeu a 328 de 471 gestantes acompanhadas, correspondendo a 69,63\% a partir do calculo fornecido pelo MS. Sendo este dado colhido através do site do DATASUS através da responsável pelo PNI da referida Secretaria de Saúde do município de estudo.

O percentual de RN com diagnóstico de sífilis congênita e tétano neonatal se apresentaram com valores zerados no primeiro semestre deste ano. Estes dados, no entanto são bastante otimistas, pois indicam a ausência destas patologias nos recém- nascidos. 


\section{Considerações finais}

Tendo em vista a constante preocupação para com a qualidade da assistência prestada as mulheres no período gestacional, os dados analisados através de pesquisas nesta área constituem-se em importante fonte para que se possam buscar mais estratégias que contribuam para uma melhora nesta assistência.

De acordo com este estudo no que tange a estrutura das unidades da zona urbana da cidade, pode-se observar que as mesmas atendem aos requisitos mínimos e considerados indispensáveis, porém há ainda o viés da frequente demanda que surge de gestantes da zona rural do município nas unidades da zona urbana, sendo que este é um ponto a ser verificado e analisado junto a ESF de origem, pois mesmo que os requisitos mínimos essenciais estejam adequados, eles podem por muitas vezes não se apresentarem em quantidades suficientes para atender a esta grande demanda, interferindo assim no atendimento. Para tanto então, há ainda a necessidade de se realizar também pesquisas com o objetivo de verificar a atual situação destas unidades da zona rural, bem como, questionando as próprias gestantes quais os fatores que a trazem a zona urbana para realizarem seu atendimento.

Porém, por mais que as unidades apresentassem com uma classificação positiva, há ainda pontos falhos em alguns quesitos, porém muitos deles não dependem dos profissionais da unidade, mas dos responsáveis pela saúde do município. Bem como, por muitas vezes informações eram dadas sem o conhecimento necessário, cabendo também aos profissionais buscarem compreender o que se passa com relação ao pré-natal, sua atual situação, o que é necessário e, sobretudo questionar quanto aos pontos falhos e buscar planejar junto aos gestores ações que possam minimizá-los.

Com relação aos indicadores, apesar de não terem como ter sido usado todos aqueles disponíveis pelo MS, os utilizados não refletiram um resultado satisfatório, o que mostra que há pontos frágeis que também necessitam de atenção e cuidado. Principalmente se o SISPRENATAL não fornece todas as informações, pois existem dados que nenhum outro sistema oferece e que há a necessidade de sempre estar a par sobre a situação do pré-natal no município. Embora o mesmo ainda esteja em aprimoramento ou adaptação, há indispensavelmente a necessidade de se ver outros meios que possam dispor informações aos profissionais responsáveis por tal assistência, bem como para que se possa estar ciente do que é falho ou não e agir diante de tais fragilidades.

Acredita-se, contudo que os objetivos da presente pesquisa foram alcançados mesmo não tendo sido realizada no município como um todo na verificação da junção da tríade estrutura, processo e resultado, sendo que neste houve somente a ausência da avaliação da estrutura das unidades da zona rural, o que ainda pode ser realizado em posteriores estudos.

Espera-se que o mesmo contribua para que todos possam ficar cientes do quanto o pré-natal é importante para a vida da gestante/concepto e o quanto este pode ser crucial no desfecho de uma 
gestação e nascimento saudáveis, pois os indicadores refletem todo o andamento da assistência prénatal em forma de números, porém cabe ainda a cada profissional buscar sempre o melhor para suas pacientes apesar de muitas vezes nem tudo ser tão favorável e nem todas as unidades serem estruturadas como assim deve ser.

Espera-se também que o presente trabalho possa servir de base para futuras pesquisas e que se possa buscar novas estratégias para melhorar esta assistência, assim como, para que se possa ampliar os conhecimentos e gerar novas formas de atuação frente às mulheres não só no período gestacional, mas também no pré-concepcional.

\section{Referências}

ANDREUCCI, C. B.; CECATTI, J. G. Desempenho de indicadores de processo do Programa de Humanização do Pré-natal e Nascimento no Brasil: uma revisão sistemática. Cad. Saúde Pública, Rio de Janeiro, v. 27, n. 6, Jun 2011.

BRASIL. Ministério da Saúde. Departamento de Atenção Básica. Atenção ao pré-natal de baixo risco. Brasília: Ministério da Saúde, 2012.a

Ministério da Saúde. Homologo a Resolução CNS Nº466, de 12 de dezembro de 2012, nos Termos do Decreto de Delegação de Competência de 12 de novembro de 1991.b

COSTA, G. R. C; CHEIN, M. B. da C; GAMA, M. E. A; COELHO, L. S. C; COSTA, A. S. V. da; CUNHA, C. L. F; BRITO, L. M.O. Caracterização da cobertura do pré-natal no Estado do Maranhão, Brasil. Rev. bras. enferm., Brasília , v. 63, n. 6, Dez. 2010.

PARIS, G. F; PELLOSO, S. M; MARTINS, P. M. Qualidade da assistência pré-natal nos serviços públicos e privados. Rev. Bras. Ginecol. Obstet., Rio de Janeiro, v. 35, n.10, Out. 2013.

POLGLIANE, R. B. S.; LEAL, M. do C.; AMORIM, M. H. C.; ZANDONADE, E.; NETO, E. T. dos S. Adequação do processo de assistência pré-natal segundo critérios do Programa de Humanização do Pré-natal e Nascimento e da Organização Mundial de Saúde. Ciênc. saúde coletiva, Rio de Janeiro , v.19, n.7, Jul 2014 .

ROCHA, R.S. Atenção pré-natal na rede básica de Fortaleza-CE: uma avaliação da estrutura, do processo e do resultado: UECE, 2011.

VETTORE, M. V; DIAS, M; VETTORE, M. V; LEAL, M. do C. Avaliação da qualidade da atenção pré-natal dentre gestantes com e sem história de prematuridade no Sistema Único de Saúde no Rio de Janeiro, Brasil. Rev. Bras. Saude Mater. Infant. Recife, v.13, n.2, Jun 2013.

ZANCHI, M; GONÇALVES, C. V; CESAR, J. A.; DUMITH, S. de C. Concordância entre informações do Cartão da Gestante e do recordatório materno entre puérperas de uma cidade brasileira de médio porte. Cad. Saúde Pública, Rio de Janeiro, v. 29, n. 5, Maio 2013. 


\section{Como citar este artigo (Formato ABNT):}

DANTAS, V.M.C.; LUNA NETO, R.T.; FREITAS, K.M.; TEIXEIRA, O.F.B.; FELIPE, F.A.; ALENCAR, A.F.O.; CRUZ, C.A.; MOURA, U.H.S.; TAVARES, N.B.F. Assistência Pré-Natal: uma avaliação do Serviço Público de Saúde no atendimento às Gestantes no Município de Icó-CE. Id on Line Revista de Psicologia, Abril de 2015, vol.9, n.26, Supl. Esp. p. 83-95. ISSN 1981-1189.

Recebido: 05/03/2015

Aceito:10/03/2015 